\title{
Alzheimer's Disease Diagnosis using Deep Learning Techniques
}

\author{
Ahmad Waleed Salehi, Preety Baglat, Gaurav Gupta
}

\begin{abstract}
Deep learning is one of the machine learning approach which has shown promising results and performance as compare to traditional algorithms of machine learning in terms of high dimensional data of MRI brain image. In this article the application of deep learning in medical field is addressed. A thorough review of various algorithms of deep learning for diagnosis of Alzheimer's disease is done, in which this disease is a progressive brain disorder that destroy the brain memory gradually, it is a common disease in older adults which is caused by dementia. It has been obtained in most research papers that the most widely used and represented algorithm is Convolutional Neural Networks (CNN) when it deals with brain image analysis. After study of various related papers for diagnosing of $A D$, we have come to this point and suggested that the $A D$ prediction at earlier stages can be increased by using an advance deep learning techniques in different dataset (ADNI, OASIS) combining to one.
\end{abstract}

Keywords: Alzheimer's disease, Deep Learning, CNN, ADNI, Neuroimaging Classification.

\section{INTRODUCTION}

\begin{abstract}
Alzheimer disease is a type and most common form of dementia that leads to neurological or brain disorder in which causes cognitive decline and progressive memory loss due to the brain cells death. Usually the symptoms in $\mathrm{AD}$ disease patient develop slowly and get severe enough that affect in their daily life. Although the main cause of this disease is not only an old age problem but also in its early stages, the memory loss is mild and their ability skills are dramatically changed. It has been expected that 1 out of 85 people in the world affect with this disease by the year 2050 [1][2]. Early diagnosis and treatment of $\mathrm{AD}$ is a possible effective treatment. Especially at an early stage of diagnosis of $\mathrm{AD}$ is challenging task. Previous studies have shown that in most AD patients the language function is lost. So usually a neuropsychological examination is used for early diagnosis of AD. The accuracy of psychological cognitive test is totally depending on the ability and experience of the clinician. Using this test with large number of AD patients will make use of more money and time. So it is important to develop automatic detection and classification method [3]. Medical
\end{abstract}

Revised Manuscript Received on February 05, 2020.

* Correspondence Author

Dr. Gaurav Gupta*, Faculty of Engineering and Technology, Shoolini University, Himachal Pradesh, India. Email: solan.gaurav@gmail.com

Preety Baglat, Faculty of Engineering and Technology, Shoolini University, Himachal Pradesh, India.

Gaurav Gupta, Faculty of Engineering and Technology, Shoolini University, Himachal Pradesh, India.

(C) The Authors. Published by Blue Eyes Intelligence Engineering and Sciences Publication (BEIESP). This is an open access article under the CC BY-NC-ND license (http://creativecommons.org/licenses/by-nc-nd/4.0/) experts are responsible for analyzing the interpretation of medical data, this is quite difficult and limited for a medical expert to interpret images because of its subjectivity and high complexity of the images, so in other areas of real world application the use of deep learning is seen as providing promising and accurate outputs for medical data. With the rapid growth of machine learning algorithm, deep learning approach has been able to classify, extract high level feature and will also help in the accurate diagnosis of AD patients with less time [4]. The first section of this article an overview of deep learning and its applications to neuroradiology is mentioned, the commonly algorithms used in deep learning for classification of Alzheimer disease with its comparison is discussed in the second section, the third section we have concluded the best fit and accurate technique for predicting $\mathrm{AD}$. And in the last we have mentioned what are the gaps and future direction.

\section{Neural network and deep learning concept:}

Machine learning is a subfield of artificial intelligence where as deep learning is a subset of machine learning. Now days, deep learning are solving many problems in every specific filed, especially in medical image analysis. It is supervised machine learning method that uses the models of deep neural networks and neuron that is the basic computational unit in a neural network and the concept of this has taken from human brain neurons. A typical deep neural network is consisting of three layers, each layers consists of nodes the first layer is the input layer, the middle is hidden layer, and the last layer is called output layer which are used for learning and analyzing the data, the more hidden layer in neural network is the more accurate detection of patterns. For an image classification, deep learning uses labeled datasets to automatically create such a classifier for $\mathrm{AD}$ prediction. A general view of neural network is depicted in figure 1, multiple signal is taken as inputs in which they are combined together in linear form then by using weights and it passes those input signals by non-linear operations to generate the output signal.

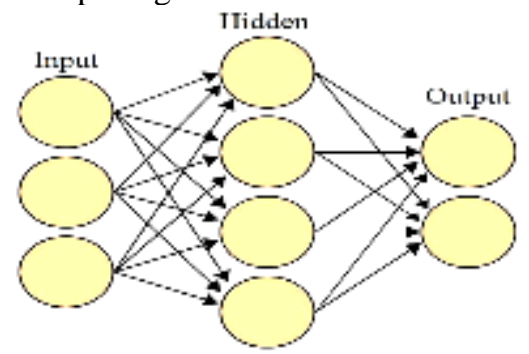

Fig 1. General view of neural network architecture[5] Deep learning is flexible approach, in which the same model can have the combination of different architecture and manipulating a range of hyper parameters [6].

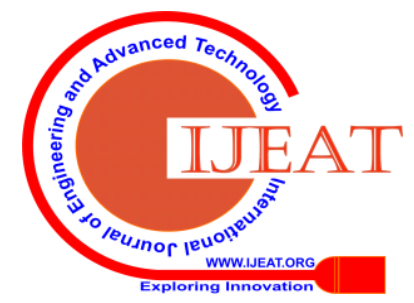


These computers aided detection and diagnosis perform by deep learning algorithms can help medical experts in interpretation of medical images, finding features and reduces the time of interpretation. Multi model neuroimaging data is used for diagnostic classification of AD in order to get better performance and a promising result [7].

\section{Deep learning methods:}

There are many deep neural network algorithms used in the area of $\mathrm{AD}$ diagnosis and which has been implemented by many researchers. These methods are more powerful as compared to other standard tools used for analyzing and learning the data.

Different architecture of neural network used in deep learning-

\section{A. Deep neural network}

It is a neural network with more than two layers one is input layer, an output layer and at least one hidden layer in between input output layer with a certain level of complex non-linear relationships. It deals with unstructured and leveled data and use for regression and classification. The architecture is shown in fig2.

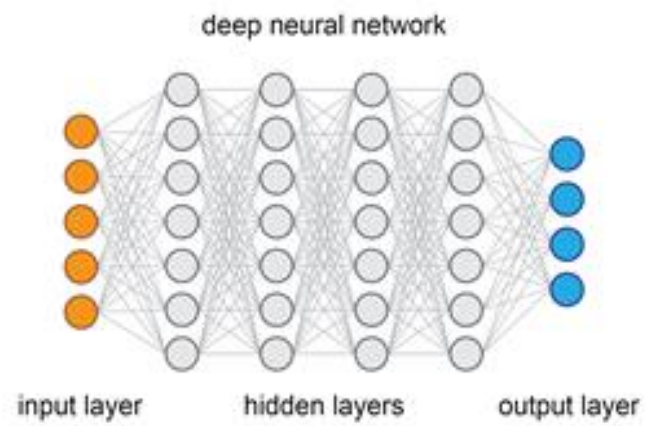

Fig 2. Deep neural network architecture

\section{B. Convolutional neural network (CNN)}

It is a type of artificial neural network are used for various classification task like image, audio etc. CNN is good for two-dimensional data. It consist of an input layer, an output layer and a hidden layer that includes multiple convolutional layers, fully connected layers, pooling layers and normalization layers. The architecture is shown in fig3.

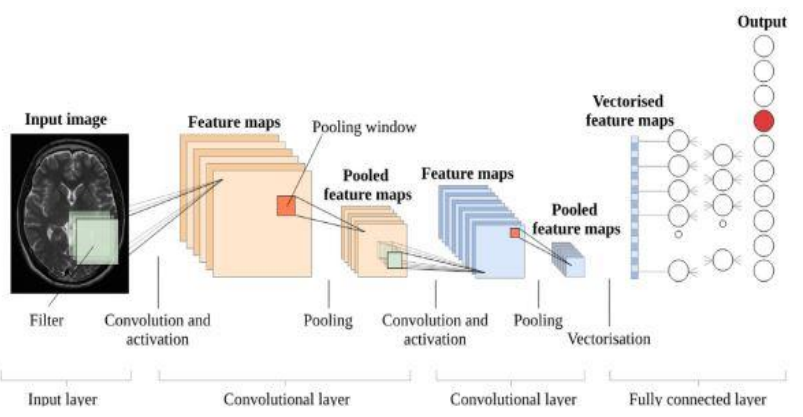

Fig 3. Architecture of convolutional neural network using medical image [8]

\section{Recurrent neural network (RNN)} natural language processing and speech recognition. In this network output from previous step are fed as input of the current step and weight are shared across all steps and
It is a type of artificial neural network commonly used in

neurons. The main feature of this network is hidden state, which has the capabilities to learn sequences. The architecture is shown in fig4.

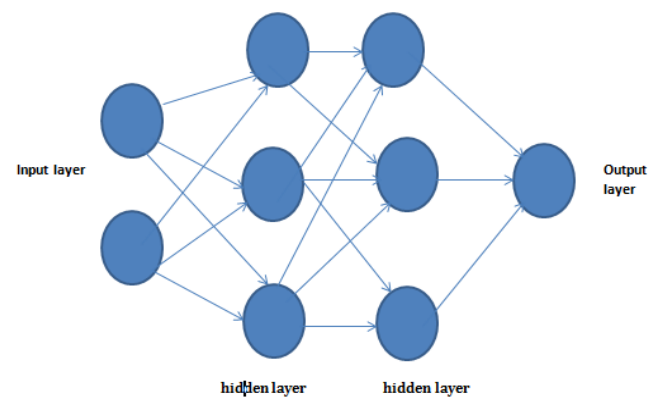

Fig 4. Recurrent neural network architecture

\section{Deep convolutional extreme learning}

This technique is the combination of CNN power and fast training of extreme learning machine and it is different from conventional neural network learning algorithms. It uses Gaussian probability and the training is faster and it efficiently completed without time- consuming iterations. This architecture is shown in fig5.

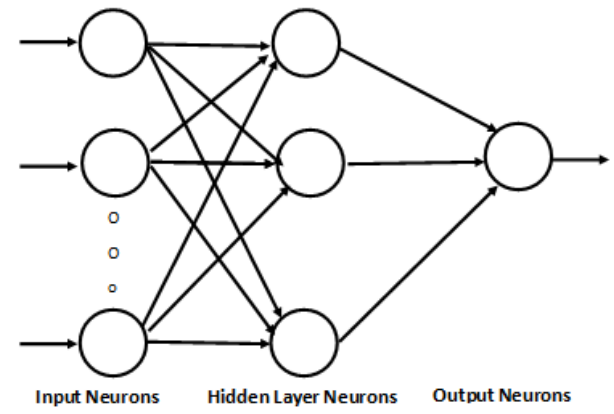

Fig

\section{DC-ELM architecture}

5.

\section{E. Deep belief network (DBN)}

It is a generative graphical models, in DBN the hidden layers are connected unidirectional and used for both supervised and unsupervised learning algorithm. Each subnetwork of the hidden layers works as a visible layer to its next layer, the DBN architecture as shown as fig 6 .

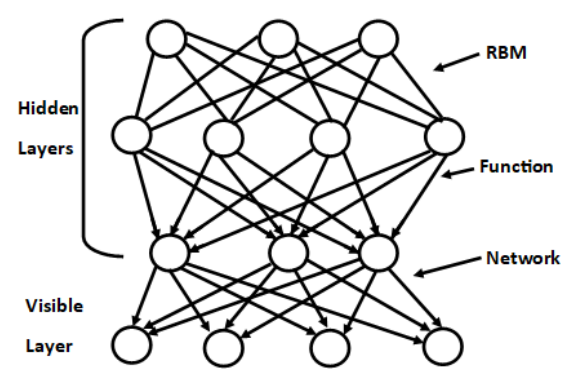

Fig 6. DBN Architecture

\section{F. Deep Autoencoder (dA)}

Deep Autoencoder is an unsupervised machine learning algorithm.

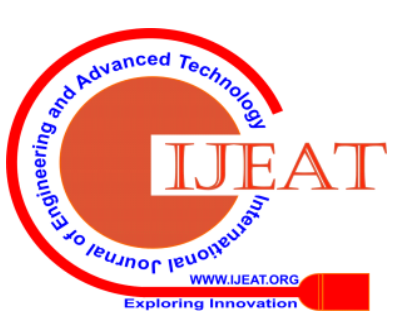


It consists of three layers input layer, hidden layer (encoding), and decoding layer. It is mostly used for extracting and redacting of dimensionality of the features architecture. The figure 7 shows the deep autoencoder and decoder in deep neural network [9]. There are basically 4 types of autoencoders such as, Denoising, Sparse, Variational, and Contractive autoencoder.

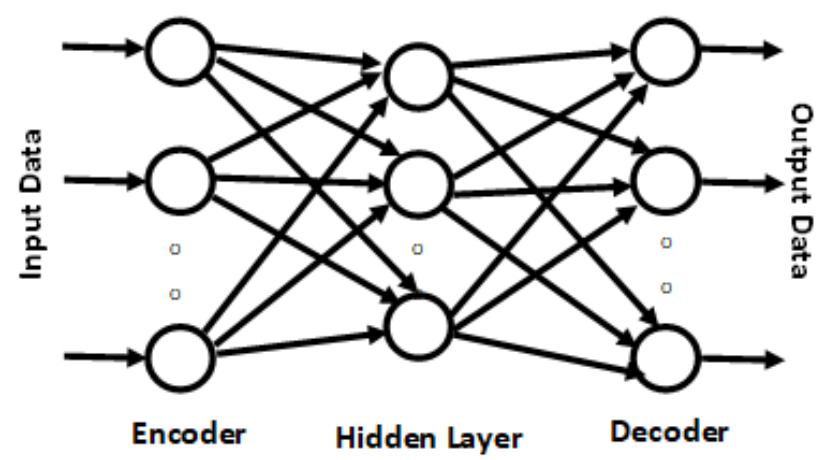

Fig 7. Architecture of an autoencoder and decoder

\section{LITERATURE SURVEY}

This section includes a brief review of the previous related paper on classification and diagnosis of $\mathrm{AD}$ and mild cognitive impairment (MIC) based on deep learning.

A novel early diagnosis technique is proposed in a paper which has used deep learning architecture for diagnosis of $\mathrm{AD}, \mathrm{MCI}$ and its earlier stage. Which contain stacked autoencoders and softmax output regression layer. This method is used to analyze multiple classes in one setting, and require less prior knowledge with minimum labeled training samples. They have taken neuroimaging data from Alzheimer's Disease Neuroimaging Initiative (ADNI). 311 subjects of MRI images from the ADNI baseline cohort, which includes 65 AD subjects, 67 cMCI (converter Mild Cognitive Impairment) subjects, 102 ncMCI (non-converters) subject and 77 normal control subjects. The results show that using both MRI and PET image they get 88.58\% accuracy for Binary classification while 47.42\% accuracy for 4-class classification while comparison was made with SK-SVM and MK-SVM. In this paper they have proven that multi-layered parametric learning can be applied on biomedical datasets with smaller size to extract high-level biomarkers [10].

S. Sarraf [11] obtained a very high accuracy of $96.85 \%$ using deep convolutional neural network architecture (LeNet-5) they have been classified functional MRI (fMRI) data of AD brain from normal healthy brain, with ADNI dataset they selected AD patients and 15 elderly normal control subjects (19 males and 24 female). And it's recommended that the more convolutional neural layer is need for complicated problems.

The same author S. Sarraf [4] in another paper using both type of data MRI and fMRI pipelines in deep convolutional neural networks, using two different subsets of ADNI dataset with 144 subjects of fMRI and 302 subjects of MRI data. In rs-fMRI data included 52 AD patients (male 31, female 21) and 92 healthy control (43 females, 49 male) and in MRI data included 211 AD (85 females, 126 male) patients and 91 healthy control subjects (43 females, male 48). The achieved the accuracy rates from both pipelines modalities and also
LeNet and GoogleNet. The accuracy rates of $99.9 \%$ and 98.84\% from both pipelines. They performed subject-level classification also for clinical purpose with $94.32 \%$ and $97.88 \%$ average accuracy rate for two different data. In case of using decision making algorithm for subject-level classification it enhanced the rate of accuracy to $97.77 \%$ (fMRI) and 100\% (MRI) pipelines.

A new technique deep learning-based technique by [12] to primary identify the mild cognitive impairment (MCI) patients with more risk to convert in to AD within 3 years. Using ADNI database they combine the base-line structural MRI, neuropsychological, demographic, and APOe4 genetic data. And it is the primary aim to discriminate an AD patient from healthy control. They achieved prediction with very high performance with an average 0.924 AUC, 86\% prediction accuracy, $87.5 \%$ of sensitivity, and $85 \%$ specificity.

As demonstrated [13] CNN based technique using structural MRI to extract its discriminative features. With the aim of the diagnosis of Early Mild Cognitive Impairment (EMCI), Late Mild Cognitive Impairment (LMCI) and classification method among healthy subjects and the two groups. They implemented on 600 subjects from ADNI database and the model for classification and feature extraction provided a high accuracy result for these three groups (EMCI, LMCI, CN (Cognitively Normal)). The best result has achieved in the sagittal view for the classification between $\mathrm{CN}$ and LMCI groups and a slightly better accuracy achieved for the EMCI/LMCI than CN/EMCI pairs concerning all views of MRI. All the mentioned results achieved below in the table from the sagittal view.

In [14] they designed and tested a pattern classification system that is combination of sparse autoencoders and 3D convolutional neural networks, in order to develop an algorithm to predict the disease status of patients. The experiments shows that a $3 \mathrm{D}$ convolutions approach in a convolutional neural network has the potential to capture local 3D patterns which may enhance the classification performance than a 2D approach. In this experiment the convolution layer is used and it is pre-trained with an autoencoder. The experiment used 22655 MRI scans from ADNI data set. And demonstrated that a 3D CNN outperform many other classifiers and produce state-of-art results.

An efficient deep convolutional neural network technique by [15] using brain MRI data analysis for diagnosis of AD and the main focus of this research work is on structured magnetic resonance image (sMRI). The model offers a significant improvement for multi-class classification, while most of existing proposed research works accomplish binary classification. It can identify different stages of $\mathrm{AD}$ and achieves higher performance in diagnosis of early stage. The training model is done on smaller dataset which is taken from OASIS dataset that contain 416 data samples.

As demonstrated [12] a multi-modal CNN framework is proposed in this paper for classification of $\mathrm{AD}$ which take the input including sMRI, clinical assessment, and genetic (APOe4) measures. They used a factorization layer approach that can retain the network depth by reducing the number of network parameter. 


\section{Alzheimer's Disease Diagnosis using Deep Learning Techniques}

This model is implemented on AD/healthy classification problem. The data has taken from ADNI1 which contain 200 NC, $400 \mathrm{MCI}$ and $200 \mathrm{AD}$ subjects in the age of between 55-90 years. The proposed framework can be able to successfully discriminate the AD patients and HCs with almost perfect accuracy.

In this paper [16] proposed a deep CNN based pipeline for diagnosis of different stages of AD using sMRI images. They implemented a 4-way classifier to classify AD, MCI, late mild cognitive impairment (LMCI) and healthy persons using ADNI dataset and total 355 volumes from 149 subjects were used. Firstly MRI scans were preprocessed to get GM images and then were passed to CNN network. The testing and training of the networks are done using ResNet and GoogLeNet models. They achieved an increased accuracy as compared to the pervious related studies.

In [17] a CNN architecture (DemNet) is proposed in this paper with modification of 16-layered VGGNet for the 3-way classification of AD, MCI and HC on MRI images which has taken from ADNI dataset. And the result outperform other classifiers from other related studies. Overall accuracy of $91.85 \%$ is achieved. It is also demonstrated in this research that 17 coronal slices from the middle part of the brain is sufficient enough for

\section{classification.}

In paper [18] they have used support vector machine (SVM) with the use of two different data sets (OASIS and ADNI). They achieved outperform results than other state-of-the-art techniques on two established data sets while using binary classification (case vs control). For experimental use we use Open Access Series of Imaging Studies $^{3}$ (OASIS) dataset and Alzheimer's Disease Neuroimaging Initiative ${ }^{2}$ (ADNI) dataset. In case of ADNI dataset they select the central part of brain with the classification results of 6 months data only and in case of OASIS dataset the use the collection of 150 subjects.

\section{RESUlt ANAlysis}

According to the study of literature survey we have compared the various techniques used for classification of $\mathrm{AD}$. As we compare the accuracy of different techniques it has shown that we can achieve better accuracy using deep convolutional neural network learning approach on MRI and fMRI dataset. The below figures shows the comparison between the techniques, modality, datasets with accuracy.

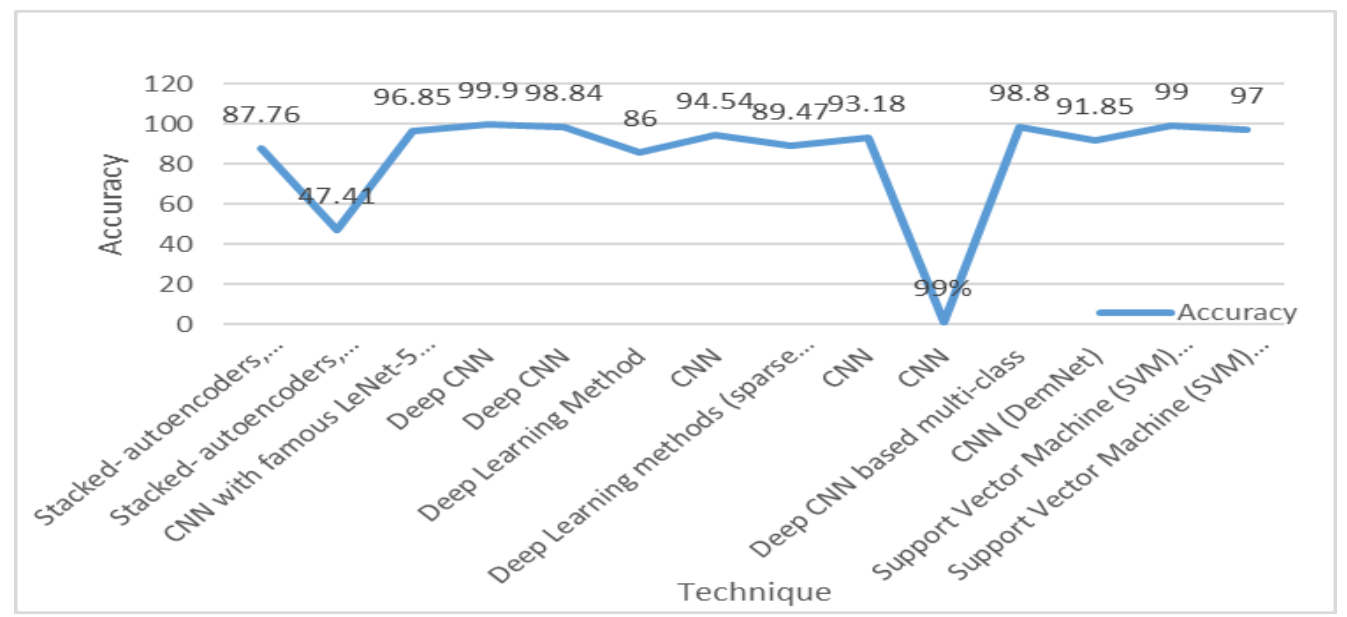

Fig 8. Comparison between techniques and accuracy

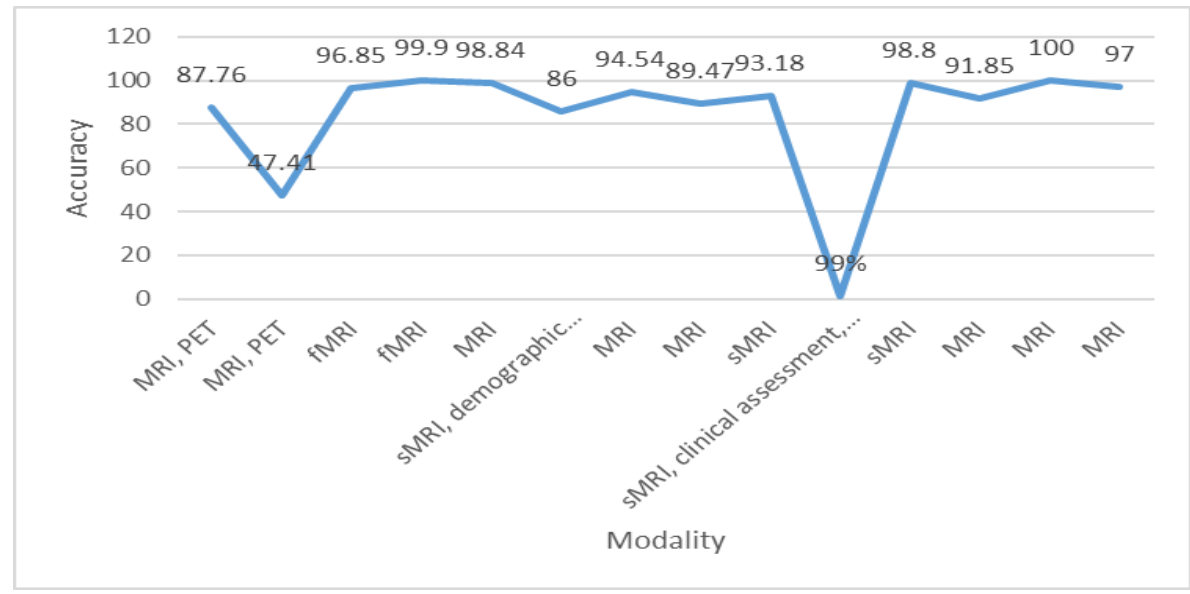

Fig 9. Comparison between modality and accuracy

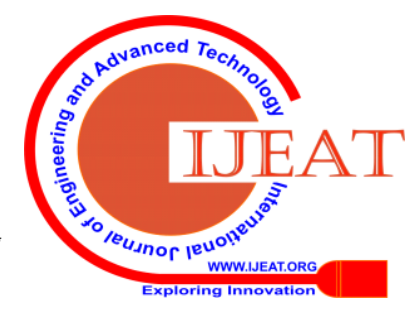




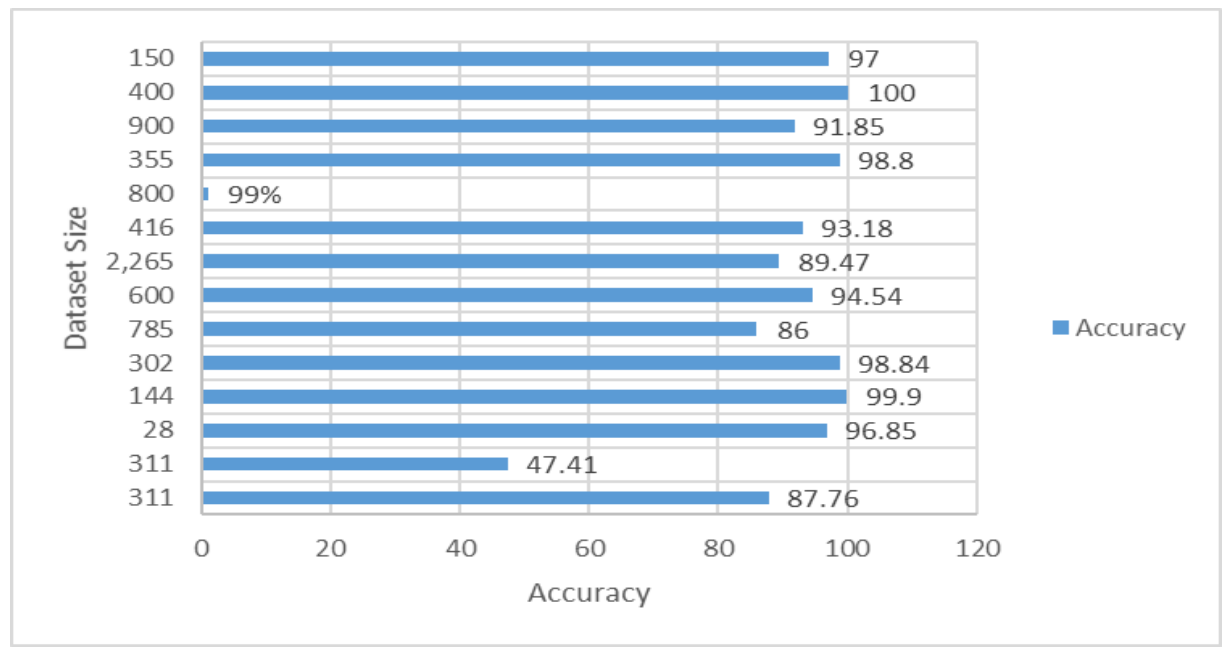

Fig 10. Comparison between dataset and accuracy

Table- I. Comparison of various technique used for classification of AD

\begin{tabular}{|c|c|c|c|c|}
\hline $\begin{array}{l}\text { Refere } \\
\text { nce }\end{array}$ & Technique & Modality & Data source type & Accuracy \\
\hline [10] & $\begin{array}{l}\text { - Stacked- } \\
\text { autoencoders } \\
\text { - Softmax- output } \\
\text { layer }\end{array}$ & MRI, PET & $\begin{array}{l}\text { ADNI- } \\
\text { MRI-311 } \\
\text { AD- 65 } \\
\text { ncMCI-102 } \\
\text { cMCI -67 } \\
\text { NC-77 }\end{array}$ & $\begin{array}{l}\text { Binary } \\
\text { classification(MRI,PET)=87.76\% } \\
\text { Four-class } \\
\text { classification(MRI,PET)=47.42\% }\end{array}$ \\
\hline [11] & $\begin{array}{l}\text { CNN with famous } \\
\text { LeNet-5 } \\
\text { Architecture }\end{array}$ & fMRI & $\begin{array}{l}\mathrm{ADNI}=28 \\
\mathrm{NC}=15 \\
\mathrm{ADNI}+\mathrm{NC}=(19 \text { male, } 24 \\
\text { female })\end{array}$ & $96.85 \%$ \\
\hline [4] & Deep CNN & MRI, fMRI & $\begin{array}{l}\text { ADNI } \\
144 \text { fMRI } \\
\text { (52 AD, } 92 \mathrm{HC}) \\
\\
302 \mathrm{MRI} \\
(211 \mathrm{AD}, 91 \mathrm{HC})\end{array}$ & $\begin{array}{l}\text { fMRI, MRI=99.9\%, 98.84\% } \\
\text { Subject-level classification- (fMRI, } \\
\text { MRI) = 94.32\%, 97.88\% } \\
\text { Using decision making- alg- (fMRI, } \\
\text { MRI) = 97.77\%, } 100 \%\end{array}$ \\
\hline [12] & $\begin{array}{l}\text { Deep Learning } \\
\text { Method }\end{array}$ & $\begin{array}{c}\text { - Base-line } \\
\text { structural MRI } \\
\text { - Demographic } \\
\text { - Neuropsychol } \\
\text { ogical } \\
\text { - APOe4 genetic } \\
\text { data }\end{array}$ & ADNI & $\begin{array}{l}\text { Predication accuracy= } 86 \% \\
\text { Sensitivity }=87.5 \% \\
\text { Specificity }=85 \%\end{array}$ \\
\hline [13] & CNN & MRI & ADNI & $\begin{array}{l}\text { Classification accuracy- } \\
\text { CN vs LMCI = 94.54\% } \\
\text { For the pairs- } \\
\text { CN/EMCI = 93.96\% } \\
\text { EMCI/LMCI = 93.00\% }\end{array}$ \\
\hline
\end{tabular}

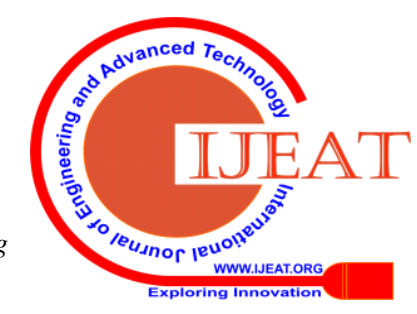


Alzheimer's Disease Diagnosis using Deep Learning Techniques

\begin{tabular}{|c|c|c|c|c|}
\hline$[14]$ & $\begin{array}{l}\text { Deep Learning } \\
\text { methods (sparse } \\
\text { autoencoders and 3D } \\
\text { CNNs) }\end{array}$ & MRI & ADNI & \begin{tabular}{lr|} 
Classification & Accuracy \\
3-way & $89.47 \%$ \\
AD vs HC & $95.39 \%$ \\
AD vs MCI & $86.84 \%$ \\
HC vs MCI & $92.11 \%$
\end{tabular} \\
\hline$[15]$ & CNN & sMRI & OASIS & $\begin{array}{l}\text { Proposed model } \\
\text { accuracy }=93.18 \% \\
\text { precision }=94 \% \\
\text { recall }=93 \% \\
\text { f1-score }=92 \%\end{array}$ \\
\hline$[12]$ & CNN & $\begin{array}{l}\text { sMRI } \\
\text { And clinical } \\
\text { assessment and } \\
\text { genetic } \\
\text { (APOe4) } \\
\text { measures. } \\
\end{array}$ & ADNI & $\begin{array}{l}\text { Average accuracy }=99 \% \\
\text { Average sensitivity }=98 \% \\
\text { Average specificity }=100 \% \\
\text { And with AUC of } 1 \text { across all test folds. }\end{array}$ \\
\hline$[16]$ & $\begin{array}{l}\text { Deep CNN based } \\
\text { multi-class }\end{array}$ & sMRI & ADNI & Accuracy $=98.8 \%$ \\
\hline [17] & CNN (DemNet) & MRI & ADNI & $\begin{array}{l}\text { 3-way classification overall accuracy= } \\
\text { 91.85\% } \\
\text { Binary classification: } \\
\text { AD vs HC }=98.33 \% \\
\text { AD vs } M C I=93.89 \% \\
\text { MCI vs HC }=91.67 \%\end{array}$ \\
\hline$[18]$ & $\begin{array}{l}\text { Support Vector } \\
\text { Machine (SVM) }\end{array}$ & MRI & ADNI, OASIS & $\begin{array}{l}\text {-Binary classification (case vs control) } \\
\text { ADNI (OASIS)- } \\
\text { Accuracy }=100 \%(97 \%) \\
\text { Sensitivity }=100 \%(97 \%) \\
\text { Specificity }=99 \%(93 \%) \\
\text {-dealing with four classes- } \\
\text { ADNI: } \\
\text { Accuracy, Sensitivity, Specificity=99\% } \\
\text { OASIS: } \\
\text { Accuracy, Sensitivity= } 77 \% \text { and } \\
\text { Specificity }=79 \%\end{array}$ \\
\hline
\end{tabular}

\section{CONCLUSION AND FUTURE SCOPE}

We have reviewed various related papers for diagnosis of Alzheimer's disease to discriminate between AD patient and healthy controls. Most of the previous research works focuses on diagnosis of $\mathrm{AD}$ and MCI based on deep learning using convolutional neural network technique and source of their data acquisition is ADNI dataset. As we studied previous research works many of the papers presented a multi-modal CNN, multi-class classification, binary classification based on deep neural network on different number of population they successfully achieved better accuracy. In this paper [11] they have used CNN deep learning architecture and famous architecture LeNet5 on fMRI data they successfully achieved the AD data from normal control with accuracy of $96.86 \%$ using ADNI date of 28 people and NC data of 15 people and the same author [4] used Deep CNN architecture but in this they used MRI and fMRI pipelines with 144 fMRI(52 AD, 92 HC) and $302 \mathrm{MRI}(211 \mathrm{AD}, 91 \mathrm{HC})$ dataset and higher result achieved 98.84\% with MRI and 99.9\% with fMRI. in [18] used the machine learning SVM techniques for classifying the $\mathrm{AD}$ patient from MRI with the use of two different data sets (OASIS and ADNI). They achieved outperform results then other state-of-the-art techniques on two established data sets while using binary classification (case vs control) with the accuracy of $100 \%$ from ADNI and $97 \%$ from OASIS dataset.

After reviewing all the paper, we concluded that in most of the papers, machine learning as well as deep learning techniques are used on separate dataset. In future work we can use advance deep learning techniques in different dataset combining to one so that efficiency and efficacy of $\mathrm{AD}$ prediction at earlier stages can be increased. 


\section{REFERENCES}

1. S. Rathore, M. Habes, M. A. Iftikhar, A. Shacklett, and C. Davatzikos, "A review on neuroimaging-based classification studies and associated feature extraction methods for Alzheimer's disease and its prodromal stages," Neuroimage, vol. 155, no. August 2016, pp. 530-548, 2017, doi: 10.1016/j.neuroimage.2017.03.057.

2. G. Gupta, A. Gupta, P. Barura, and V. Jaiswal, "Mobile Health Applications and Android Toolkit for Alzheimer Patients , Caregivers and Doctors Mobile Health Applications and Android Toolkit for Alzheimer Patients , Caregivers and Doctors," no. July, pp. 198-205, 2019.

3. G. Lyu, “A Review of Alzheimer's Disease Classification Using Neuropsychological Data and Machine Learning," Proc. - 2018 11th Int. Congr. Image Signal Process. Biomed. Eng. Informatics, CISP-BMEI 2018, no. 2017, pp. 1-5, 2019, doi 10.1109/CISP-BMEI.2018.8633126.

4. S. Sarraf, D. D. DeSouza, J. Anderson, G. Tofighi, and for the A. D. N. Initiativ, "DeepAD: Alzheimer's Disease Classification via Deep Convolutional Neural Networks using MRI and fMRI," bioRxiv, no. August 2016, p. 070441, 2017, doi: 10.1101/070441.

5. M. I. Razzak, S. Naz, and A. Zaib, "Deep learning for medical image processing: Overview, challenges and the future," Lect. Notes Comput. Vis. Biomech., vol. 26, no. August, pp. 323-350, 2018, doi: 10.1007/978-3-319-65981-7_12.

6. S. Vieira, W. H. L. Pinaya, and A. Mechelli, "Neuroscience and Biobehavioral Reviews Using deep learning to investigate the neuroimaging correlates of psychiatric and neurological disorders: Methods and applications," Neurosci. Biobehav. Rev., vol. 74, pp. 58-75, 2017, doi: 10.1016/j.neubiorev.2017.01.002.

7. T. Jo, K. Nho, and A. J. Saykin, "Deep Learning in Alzheimer' s Disease: Diagnostic Classification and Prognostic Prediction Using Neuroimaging Data," vol. 11, no. August, 2019, doi: 10.3389/fnagi.2019.00220.

8. A. S. Lundervold and A. Lundervold, "An overview of deep learning in medical imaging focusing on MRI," Z. Med. Phys., vol. 29, no. 2, pp. 102-127, 2019, doi: 10.1016/j.zemedi.2018.11.002.

9. T. Ma, F. Wang, J. Cheng, Y. Yu, and X. Chen, "A hybrid spectral clustering and deep neural network ensemble algorithm for intrusion detection in sensor networks," Sensors (Switzerland), vol. 16, no. 10, 2016, doi: 10.3390/s16101701.

10. S. Liu, S. Liu, W. Cai, S. Pujol, R. Kikinis, and D. Feng, "Early diagnosis of Alzheimer's disease with deep learning," 2014 IEEE 11th Int. Symp. Biomed. Imaging, ISBI 2014, pp. 1015-1018, 2014, doi: 10.1109/isbi.2014.6868045

11. S. Sarraf and G. Tofighi, "Classification of Alzheimer's Disease Structural MRI Data by Deep Learning Convolutional Neural Networks," pp. 8-12, 2016.

12. S. Spasov, L. Passamonti, A. Duggento, P. Liò, and N. Toschi, "A parameter-efficient deep learning approach to predict conversion from mild cognitive impairment to Alzheimer's disease," Neuroimage, vol. 189, no. August 2018, pp. 276-287, 2019, doi: 10.1016/j.neuroimage.2019.01.031.

13. H. T. Gorji and N. Kaabouch, "brain sciences A Deep Learning approach for Diagnosis of Mild Cognitive Impairment Based on MRI Images," 2019.

14. A. Payan and G. Montana, "Predicting Alzheimer's disease a neuroimaging study with 3D convolutional neural networks," ICPRAM 2015 - 4th Int. Conf. Pattern Recognit. Appl. Methods, Proc., vol. 2, pp. 355-362, 2015

15. J. Islam and Y. Zhang, "Brain MRI analysis for Alzheimer's disease diagnosis using an ensemble system of deep convolutional neural networks," Brain Informatics, vol. 5, no. 2, 2018, doi: 10.1186/s40708-018-0080-3.

16. A. Farooq, S. Anwar, M. Awais, and S. Rehman, "A deep CNN based multi-class classification of Alzheimer's disease using MRI," IST 2017 IEEE Int. Conf. Imaging Syst. Tech. Proc., vol. 2018-Janua, pp. 1-6, 2017, doi: 10.1109/IST.2017.8261460.

17. C. D. Billones, O. J. L. D. Demetria, D. E. D. Hostallero, and P. C. Naval, "DemNet: A Convolutional Neural Network for the detection of Alzheimer's Disease and Mild Cognitive Impairment," IEEE Reg. 10 Annu. Int. Conf. Proceedings/TENCON, pp. 3724-3727, 2017, doi: 10.1109/TENCON.2016.7848755.

18. F. Previtali, P. Bertolazzi, G. Felici, and E. Weitschek, "A novel method and software for automatically classifying Alzheimer's disease patients by magnetic resonance imaging analysis," Comput. Methods Programs Biomed., vol. 143, pp. 89-95, 2017, doi: 10.1016/j.cmpb.2017.03.006.

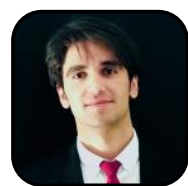

\section{AUTHORS PROFILE}

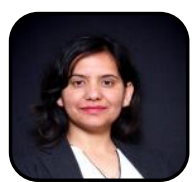

Ahmad Waleed Salehi, received BCS from Rana University, currently student of MTech CSE in Shoolini University, Himachal Pradesh, India.

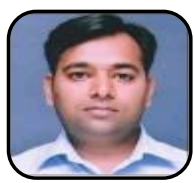

(a) Gupta, received BE degree from Dr. B. R. Ambedkar University, Agra, UttarPradesh. He received PG and Ph.D. degree in the stream of Computer Science Engineering from KSOU-VIT, Karnataka and Shoolin University respectively. He attended more number of conferences and presented more papers in the field of machine learning and artificial intelligence. Currently he is working as an Assistant professor in School of Electrical and Computer Science Engineering, Shoolini University, Solan, Himachal Pradesh. His area of interest is in the field of Machine Learning, Big data, Cloud Computing and Databases. 